\title{
Structural Analysis and Size Optimization of a Fine-Blanking Press Frame Based on Sensitivity Analysis
}

\author{
Xinhao Zhao1,3 - Yanxiong Liu2,3,* - Lin Hua2,3 - Huajie Mao1,3 \\ ${ }^{1}$ Wuhan University of Technology, School of Materials Science and Engineering, China \\ 2 Wuhan University of Technology, Hubei Collaborative Innovation Center for Automotive Components Technology, China \\ ${ }^{3}$ Wuhan University of Technology, Hubei Key Laboratory of Advanced Technology for Automotive Components, China
}

The fine-blanking press, which has been widely used in the sheet-cutting industry, is high-end equipment. The frame is a core component of the fine-blanking press. In this paper, the sensitivity analysis and size optimization method were used to optimize a fine-blanking press frame. First, a shell element model of the frame was established to analyse the mechanical properties of the initial frame. Then, the mathematical expressions for sensitivity analysis and size optimization of the frame were formulated. Based on the established model, the response sensitivities for plate thicknesses were analysed, and a $12.94 \%$ weight reduction was achieved through size optimization. Moreover, the effect of material distribution on dynamic performance was investigated. The result indicated that for a constrained structure, distributing material close to the constraint location increases the modal frequencies.

Keywords: fine-blanking press frame; optimal design; sensitivity analysis; size optimization; dynamic performance

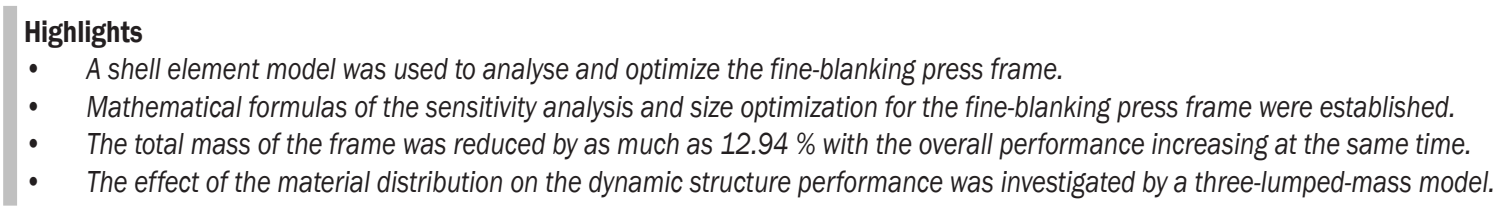

\section{INTRODUCTION}

Fine blanking is a specialized form of blanking, which can produce parts with full clean-cut surfaces only in one operation. Fine blanking has been widely used in many industrial fields because of its high efficiency and high precision. Taking the vehicle production as an example, there are almost 100 to 200 fine-blanking products in a car [1]. The fineblanking process is achieved in a specialized fineblanking press, which has a few critical additional parts compared to the traditional blanking press and can provide hydrostatic compressive stress. Generally, elastic deformation and vibration of the press are considered undesirable in the fine-blanking process. A fine-blanking press frame is a structure that bears immense pressure and high excitation frequencies during the fine-blanking process as well as supports many other crucial components and systems. The traditional structural design of the frame mostly relies on experience and simple calculation, which can ensure the mechanical properties but also increase the materials consumption. Allwood et al. calculated that the production of mechanical equipment consumes about $13 \%$ of all the steel produced in the world and a non-negligible amount of cast iron and aluminium [2]. Weight reduction is an effective way to reduce the materials and energy consumption in manufacture and transportation area. Hence, the challenge in designing a fine-blanking press frame is in synthesizing ideal and lightweight structural properties. The following aspects must be considered during the frame optimization design: required global and local static stiffness, sufficient structure strength, desired dynamic property, and minimized weight or mass.

The problem of design optimization, especially structural optimization, has been and will continue to be an area of much research interest. The main research interests include the most popular solid isotropic material with penalization (SMIP) method [3] and [4], level set method [5] and [6], evolutionary structural optimization (ESO) method [7] and [8], and size and shape optimization technologies [9] to [11], etc. Due to the maturity of these optimization techniques, they have been widely used for lightweight purposes considering the overall performance of a structure. The most commonly used optimization design procedures are combinations of topology optimization, size optimization, and shape optimization [12] to [14]. In these research studies, topology optimization was conducted in the conceptual design stage to distribute the material in the design domain, and size and shape optimization were then performed in the detailed design stage to minimize the structure weight further. Structural sensitivity is another significant parameter. With the use of sensitivity analysis, the most 
efficient variables can be identified and optimized systematically. Many researchers have also made use of the sensitivity information of a structure to improve its performance and reduce its weight [15] to [17].

In recent years, finite element analysis, experimental studies and the mentioned optimization methods (e.g., topology optimization, shape optimization, size optimization, and sensitivity analysis, etc.) have been conducted in investigating and optimizing presses and press frames. Strano et al. researched the optimization of pre-stressed press frames [18] and conducted an optimized design of press frames with respect to energy efficiency [2]. Trebuňa et al. discussed the fatigue crack growth in the press frame and gave out solutions to suppress crack initiation [19]. Zhang et al. introduced the cylinder-crown integrated hydraulic press (CCIHP), and finite element analysis for CCIHP showed that both the stress and displacements on the press during the loading process are allowable, even if eccentric force is loaded [20]. Wang et al. proposed a novel pre-stressed wire-wound orthogonal preload frame structure for multi-directional forging press to deal with the contradiction between the independence of structure and the independence of mechanics [21]. Glebov et al. applied the most common topology optimization methods (ESO and SIMP) to the optimal design of the traverse of a column press, and the comparison between the optimized structure and the existing traverse was presented [22].

Concerning fine-blanking press frame, Lan et al. optimized the fine-blanking press frame by applying topology and shape optimization [23]. Unfortunately, the optimization result featured with material stacks was difficult to be interpreted into a frame structure welded by thick plates. To deal with this problem, Zhao et al. proposed a two-stage topology optimization procedure to optimize the fine-blanking press frame, which was proved to be appropriate and feasible [24]. However, the interpreted structure was irregular and complicated, which was difficult for manufacturing and transporting.

This study aims to seek a new optimization method for the existing regular-shaped frame structure. Sensitivity analysis and size optimization methods were used to analyse and optimize the frame. In this work, the shell element model was used to simulate the frame. The response sensitivities with respect to plate thicknesses were analysed. The frame weight was reduced by as much as $12.94 \%$ through size optimization. In addition, the influence of material distribution on dynamic performance of a constrained structure was discussed, and the result indicated that material distributing close to the constraint location makes for the increasing of the modal frequencies.

The subsequent sections are organized as follows. In Section 1, the shell element model of the initial fine-blanking press frame was established, and the mechanical properties were analysed by finite element analysis. Section 2 states the mathematical formulas of the frame sensitivity analysis and size optimization. Section 3 shows the sensitivity information and optimization result of the frame and discusses the influence of material distribution on the dynamic performance of a constrained structure. Section 4 summarizes the study.

\section{FINITE ELEMENT ANALYSIS OF THE FINE-BLANKING PRESS FRAME}

\subsection{Finite Element Modelling of the Initial Frame}

As shown in Fig. 1, a 12,000 kN fine-blanking press frame, which was produced by Huangshi Huali Metal Forming Machine Tool Inc., was welded together by several Q235-A plates with different thicknesses. The frame was 2,800 $\mathrm{mm}$ long, 2,000 $\mathrm{mm}$ wide, and 4,500 $\mathrm{mm}$ high. The frame bears pressure from other components and guides the moving slider during the fine-blanking process, and to ensure the blanking accuracy, the deformation of load-bearing surfaces and guiding surfaces (shown in Fig. 1) should be within a certain range.

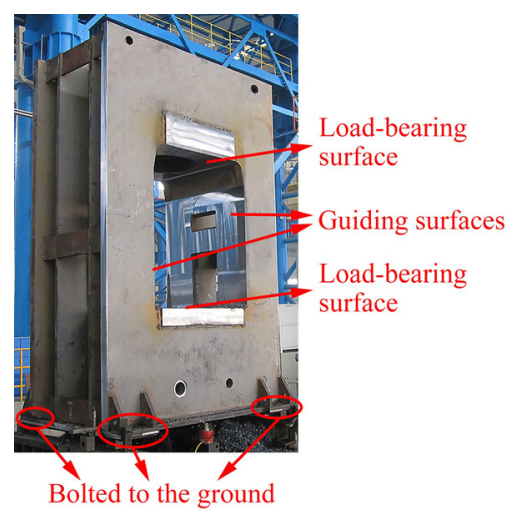

Fig. 1. Photograph of the $12,000 \mathrm{kN}$ fine-blanking press frame

Fig. 2 shows the modelling of the frame. The plates were classified into 15 groups according to their thicknesses, locations, and shapes (Fig. 2a). Wang and Mai [25] determined that the basic principle of using shell element is that the main size of plate should not be less than 10 times its thickness, which is met by almost all the frame plates. Therefore, to conduct plate 
thickness sensitivity analysis and size optimization, the finite element model of the frame was established using shell elements (Fig. 2b). The model was built up by adopting Altair Hypermesh. The average size of the element is $20 \mathrm{~mm}$. The model contains 145,688 quad 4 elements, 1,000 tria 3 elements, 1 rbe 3 element, 2 rigidlink (rbe2) elements and 146,640 nodes. A linear elastic material model was used, and Q235-A was considered with the following properties: Young's modulus $E$ was $212 \mathrm{GPa}$, Poisson ratio $v$ was 0.288 , and mass density $\rho$ was $7.86 \times 10^{-6} \mathrm{~kg} / \mathrm{mm}^{3}$. Constraints were applied on the nodes that site in the bolt hole (all DOFs restrained) and the surfaces contacted with the ground (only the DOFs of Z-direction displacement were restrained). A previous work [24] compared two kinds of loading methods for the fine-blanking press frame: 1) forces were uniformly distributed to the load-bearing nodes and 2) the loaded surfaces were assumed as rigid surfaces, on which a concentrated force was applied. The simulation result showed that the latter is more consistent with the experiment result. Hence, in this study, the load-bearing nodes were connected by an RBE 2 element, which is a kind of rigid element, and a concentrated force was applied directly on the master node. Furthermore, the guiding surface nodes were connected by a flexible RBE 3 element, whose nodal displacement represented the average deformation of the guiding surface (only one guiding surface was considered because of symmetry).

\subsection{Finite Element Analysis Results}

The finite element analysis was conducted using Altair Radioss. The total mass $M$, maximum stress $\sigma_{\max }$, compliance $c$, displacements of node 1 in $\mathrm{Z}$ direction $d_{1 z}$, node 2 in $\mathrm{Z}$ direction $d_{2 z}$ and node 3 in
Y direction $d_{3 y}$ (node number illustrated in Fig. 2) and the first-order modal frequency $f_{1}$ were obtained and exhibited in Table 1.

Table 1. Finite element analysis results of the initial frame

\begin{tabular}{ccccccc}
\hline $\begin{array}{c}M \\
{[\mathrm{~kg}]}\end{array}$ & $\begin{array}{c}\sigma_{\max } \\
{[\mathrm{MPa}]}\end{array}$ & $\begin{array}{c}c \\
{[\mathrm{~kJ}]}\end{array}$ & $\begin{array}{c}d_{1 z} \\
{[\mathrm{~mm}]}\end{array}$ & $\begin{array}{c}d_{2 z} \\
{[\mathrm{~mm}]}\end{array}$ & $\begin{array}{c}d_{3 y} \\
{[\mathrm{~mm}]}\end{array}$ & $\begin{array}{c}f_{1} \\
{[\mathrm{~Hz}]}\end{array}$ \\
\hline 40740.50 & 123.4 & 2.471974 & 0.116 & 0.296 & 0.08673 & 41.04213 \\
\hline
\end{tabular}

\section{ESTABLISHMENT OF THE SENSITIVITY ANALYSIS AND SIZE OPTIMIZATION MODELS}

\subsection{Sensitivity Analysis Model}

This section introduces the analytical sensitivity expressions for the response quantities (total mass $M$, compliance $c$, nodal displacements $d_{1 z}, d_{2 z}$, and $d_{3 y}$ and first-order modal frequency $f_{1}$ ) with respect to the design variables (plate thicknesses $T_{k}$ ). According to the engineering experience, when designing a fine-blanking press frame, the stiffness requirement is always more critical than the structure strength requirement, and the structural strength is always satisfied when the stiffness is satisfied. Therefore, the maximum stress $\sigma_{\max }$ was only used for structure strength checking.

\subsubsection{Total Mass Sensitivity}

The total mass of the frame structure $M$ is an implicit function of the plate thicknesses, i.e.,

$$
M=\sum_{k=1}^{15} \rho A_{k} T_{k},
$$

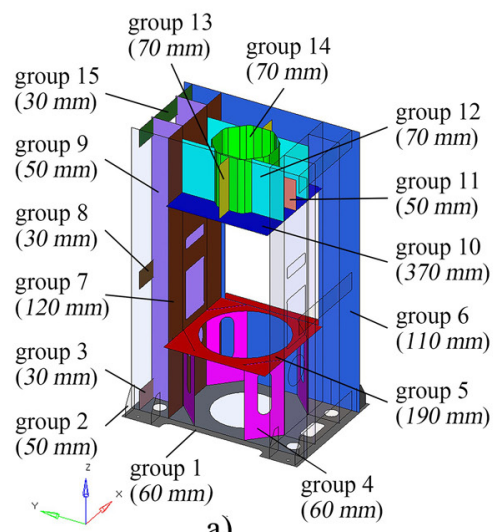

a)

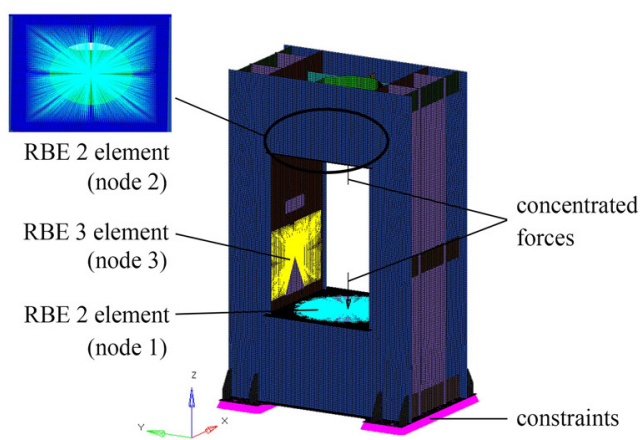

b)

Fig. 2. Frame modelling: a) plate classifying and initial thickness of each group, and b) finite element model of the frame 
where $\rho$ is the density of the frame, $A_{k}$ is the total plate area of the $k^{\text {th }}$ group, and $T_{k}$ is the thickness of the $k^{\text {th }}$ group ( $1 \leq k \leq 15$, similarly hereinafter).

Taking derivative of Eq. (1) with respect to the plate thickness, the mass sensitivity $S_{M}$ can be obtained as,

$$
S_{M}=\frac{\partial M}{\partial T_{k}}=\rho A_{k}
$$

\subsubsection{Total Mass Sensitivity}

In practice, the deformation of loaded surfaces and guiding surfaces of the fine-blanking press frame are concerned. Hence, in this research, the displacements of node 1 in $\mathrm{Z}$ direction $d_{1 z}$, node 2 in $\mathrm{Z}$ direction $d_{2 z}$ and node 3 in $\mathrm{Y}$ direction $d_{3 y}$ were adopted to estimate the local static stiffness. Furthermore, the global static stiffness of the frame is measured by the compliance $c$, which is the strain energy of the structure and can be considered a reciprocal measure for the stiffness of the structure [26]. In general, the smaller the compliance is, the higher the global static stiffness is.

Given the equation of motion,

$$
\mathbf{K U}=\mathbf{P},
$$

and its derivative with respect to design variable $T_{k}$ is,

$$
\frac{\partial \mathbf{K}}{\partial T_{k}} \mathbf{U}+\mathbf{K} \frac{\partial \mathbf{U}}{\partial T_{k}}=\frac{\partial \mathbf{P}}{\partial T_{k}},
$$

where $\mathbf{K}$ is the global stiffness matrix, $\mathbf{U}$ and $\mathbf{P}$ are the vectors of nodal displacement and force, respectively. Then, considering $\mathbf{P}$ is a constant, the displacement derivative with respect to $T_{k}$ can be obtained as,

$$
\frac{\partial \mathbf{U}}{\partial T_{k}}=-\mathbf{K}^{-1} \frac{\partial \mathbf{K}}{\partial T_{k}} \mathbf{U}
$$

Based on Eq. (5), the concerned local stiffness sensitivities $S_{d 1 z}, S_{d 2 z}$ and $S_{d 3 y}$ can be obtained.

The frame compliance $c$ is the function of nodal displacement and load vectors,

$$
c=\frac{1}{2} \mathbf{U}^{T} \mathbf{P} .
$$

Taking the derivative of Eq. (6) with respect to $T_{k}$ and uniting Eq. (3) and Eq. (5), the global static stiffness sensitivity $S_{c}$ can be deduced as,

$$
S_{c}=\frac{\partial c}{\partial T_{k}}=\frac{1}{2} \frac{\partial \mathbf{U}^{T}}{\partial T_{k}} \mathbf{P} .
$$

\subsubsection{Dynamic Stiffness Sensitivity}

Dynamic stiffness is commonly evaluated by the structural modal frequencies. The eigenvalue equation of the frame free vibration is:

$$
\left(\mathbf{K}-\omega_{i}^{2} \mathbf{M}\right) \mathbf{u}_{i}=\mathbf{0},
$$

where $\mathbf{M}$ is the global mass matrix, $\omega_{i}$ is the $i^{\text {th }}$ order modal circular frequency, which is $2 \pi$ times as large as the $i^{\text {th }}$ order modal frequency $f_{i}$, and $\mathbf{u}_{i}$ is the $i^{\text {th }}$ order mode shape.

The derivative of Eq. (8) with respect to design variable $T_{k}$ is:

$$
\begin{gathered}
\left(\frac{\partial \mathbf{K}}{\partial T_{k}}-2 \omega_{i} \frac{\partial \omega_{i}}{\partial T_{k}} \mathbf{M}-\omega_{i}^{2} \frac{\partial \mathbf{M}}{\partial T_{k}}\right) \mathbf{u}_{i} \\
+\left(\mathbf{K}-\omega_{i}^{2} \mathbf{M}\right) \frac{\partial \mathbf{u}_{i}}{\partial T_{k}}=\mathbf{0},
\end{gathered}
$$

then, by uniting Eq. (9) and $\omega_{i}=2 \pi f_{i}$, we can obtain the dynamic response sensitivity $S_{f i}$ :

$$
S_{f i}=\frac{\partial f_{i}}{\partial T_{k}}=\frac{1}{4 \pi \omega_{i}} \mathbf{u}_{i}^{T}\left(\frac{\partial \mathbf{K}}{\partial T_{k}}-\omega_{i}^{2} \frac{\partial \mathbf{M}}{\partial T_{k}}\right) .
$$

\subsection{Size Optimization Model}

A size optimization method was used to optimize the $12,000 \mathrm{kN}$ fine-blanking press frame. An alternative way to conduct optimization is a multi-objective optimization method, in which the volume fraction is chosen as a constraint condition, and both static and dynamic responses are regarded as objectives. The multi-objective can be converted into a singleobjective by adopting compromise programming [27] and [28]. With this method, not all criteria can be satisfied simultaneously while the compromised objective can reach an ideal point. Therefore, the multi-objective optimization method is suitable for optimization problems that have specific volume fraction requirement, while the static and dynamic criteria are not specific. The frame optimization problem in this paper has specific static and dynamic criteria, and the volume fraction requirement is not specific. Therefore, the multi-objective optimization procedure is not suitable.

In this study, the objective was to minimize the total mass of the frame. The thicknesses of the plates were chosen as design variables, and the constraints included compliance, displacements of loaded and guiding surfaces, and the first-order modal frequency. The optimization problem can be mathematically stated as: 
Find: $\quad T_{1}, T_{2}, T_{3}, \ldots, T_{15}$

Minimize: $M\left(T_{1}, T_{2}, T_{3}, \ldots, T_{15}\right)$

$$
\text { Subject to }:\left\{\begin{array}{l}
\mathbf{K U}=\mathbf{P}, \\
d_{1 z} \leq D_{1 z}, d_{2 z} \leq D_{2 z}, \\
d_{3 y} \leq D_{3 y} ; \\
c=\frac{1}{2} \mathbf{U}^{T} \mathbf{P}=\frac{1}{2} \mathbf{U}^{T} \mathbf{K} \mathbf{U}, \\
c \leq C ; \\
\omega_{i}{ }^{2}=\frac{K_{i}}{M_{i}}=\frac{\Phi_{i}{ }^{T} \mathbf{K} \Phi_{i}}{\Phi_{i}{ }^{T} \mathbf{M} \Phi_{i}}, \\
\omega_{i}=2 \pi f_{i}, f_{1} \geq F_{1} ; \\
T_{k \min } \leq T_{k} \leq T_{k \max } \\
(1 \leq k \leq 15) .
\end{array}\right.
$$

where $K_{i}$ and $M_{i}$ are the $i^{\text {th }}$ order modal stiffness and modal mass, respectively, $\boldsymbol{\Phi}_{i}$ is the eigenvector of the $i^{\text {th }}$ order mode of vibration, $C$ is the upper bound for $c, D_{1 z}, D_{2 z}$ and $D_{3 y}$ are the upper bound for $d_{1 z}$, $d_{2 z}$ and $d_{3 y}$, respectively, $F_{1}$ is the lower bound for $f_{1}$, $T_{k \min }$ and $T_{k \max }$ are the lower and upper limits for $T_{k}$, respectively. According to the finite element analysis result in Section 2.2, the values of $C, D_{1 z}, D_{2 z}, D_{3 y}$, and $F_{1}$ are chosen as follows: $C=2.195 \mathrm{~kJ}, D_{1 z}=0.0892$ $\mathrm{mm}, D_{2 z}=0.276 \mathrm{~mm}, D_{3 y}=0.0697 \mathrm{~mm}$, and $F_{1}=40$ Hz. The values of $T_{k \min }$ and $T_{k \max }$ are shown in Table 2. The optimization problem was solved by adopting the Altair Optistruct, which can provide an efficient and credible finite element-based structural optimization procedure [26].

Table 2. The values of $T_{k \min }$ and $T_{k \max }$

\begin{tabular}{lcccccccc}
\hline & \multicolumn{8}{c}{ Group No. } \\
\cline { 2 - 8 } & 1 & 2 & 3 & 4 & 5 & 6 & 7 & 8 \\
\hline$T_{k \min }[\mathrm{mm}]$ & 5 & 5 & 5 & 5 & 5 & 5 & 5 & 5 \\
\hline$T_{k \max }[\mathrm{mm}]$ & 200 & 100 & 100 & 120 & 300 & 200 & 200 & 100 \\
\hline & 9 & \multicolumn{7}{c}{ Group No. } \\
& 9 & 10 & 11 & 12 & 13 & 14 & 15 \\
\hline$T_{k \min }[\mathrm{mm}]$ & 5 & 5 & 5 & 5 & 5 & 5 & 5 \\
\hline$T_{k \max }[\mathrm{mm}]$ & 100 & 400 & 200 & 200 & 100 & 200 & 100 \\
\hline
\end{tabular}

\section{SENSITIVITY ANALYSIS AND SIZE OPTIMIZATION RESULT}

\subsection{Sensitivity Analysis Results}

After carrying out the sensitivity analysis, the concerned sensitivity information is shown in Fig. 3 in column bars. It can be seen from Figs. 3 a to e that $M$ is most sensitive to $T_{6}$, followed by $T_{7}$ and $T_{9} ; c$ and $d_{2 z}$ are most sensitive to $T_{7}$, and second and third sensitive to $T_{6}$ and $T_{9}$, respectively; $d_{1 z}$ is most sensitive to $T_{5}$, followed by $T_{7}, T_{6}$, and $T_{4} ; d_{3 y}$ is most and second sensitive to $T_{7}$ and $T_{6}$, respectively. Therefore, we can reduce the total mass by appropriately decreasing $T_{6}$ and increasing $T_{5}$ and $T_{7}$ without static stiffness degradation. From Fig. 3f, it can be observed that $S_{f 1}$ of the lower part plates (groups 1 to 5) are positive, and $S_{f 1}$ of the upper part plates (groups 10 to 15) are negative, which indicates that $S_{f 1}$ can be improved by increasing the lower frame mass and decreasing the upper frame mass. This phenomenon will be discussed in detail in the following sections.

\subsection{Size Optimization Results}

Size optimization was conducted to optimize the plate thicknesses, and the thicknesses of plates were finally designed according to the optimized result. The initial, optimized and finally designed thicknesses are shown in Table 3, and the performance comparisons before and after optimization are summarized in Table 4.

Table 3. Initial, optimized and finally designed thicknesses

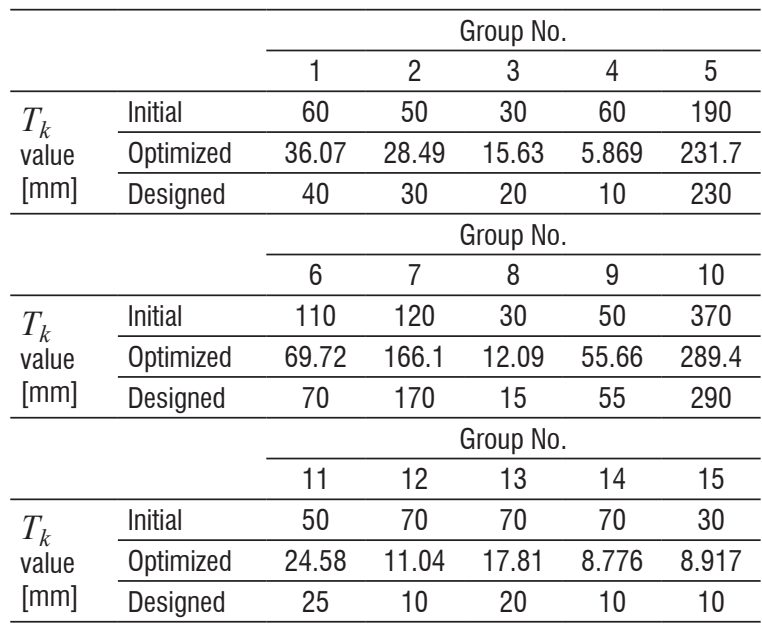

The analysis and conclusions of the previous section are verified by the modified frame. According to the analysis result, the total frame mass $M$ is reduced by $12.94 \%$. Meanwhile, the following conclusions can be drawn: the maximum stress $\sigma_{\max }$ decreases $14.62 \%$, which means that the structure strength has been improved; the compliance $c$ decreases $2.81 \%$, which indicates that the global stiffness has been improved; the node displacements $d_{1 z}, d_{2 z}$, and $d_{3 y}$ are reduced by $7.62 \%, 1.37 \%$, and $2.05 \%$, respectively, which means that the local stiffness increased; the first-order modal frequency $f_{1}$ is increased by $1.03 \%$, 


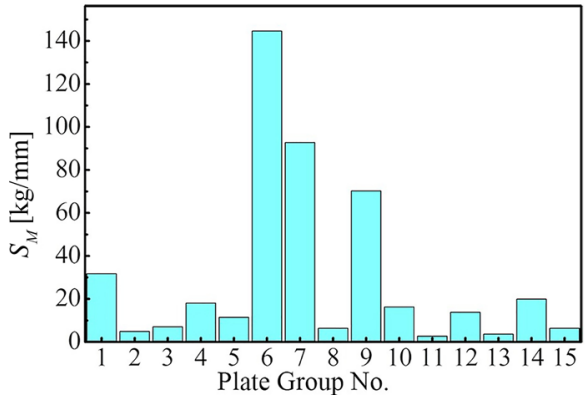

a)

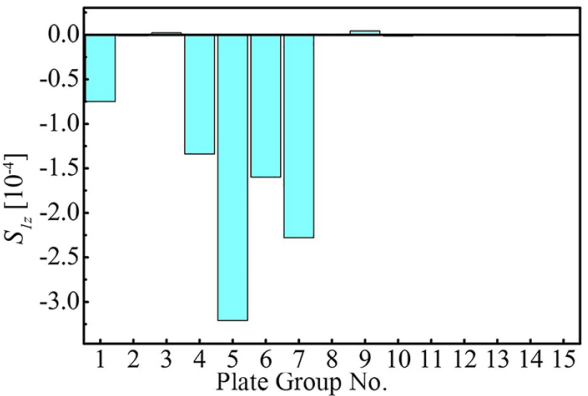

c)

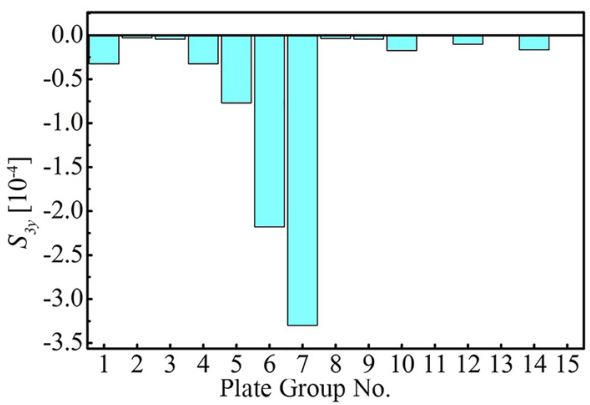

e)

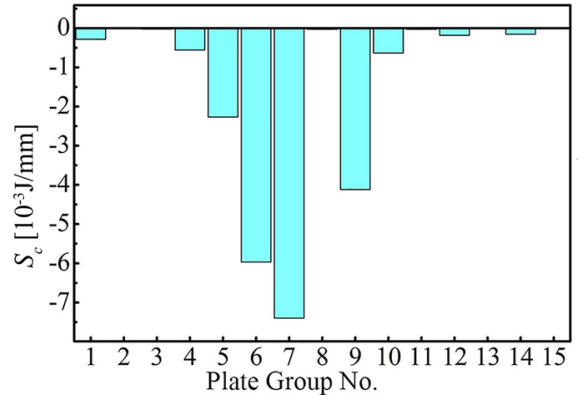

b)

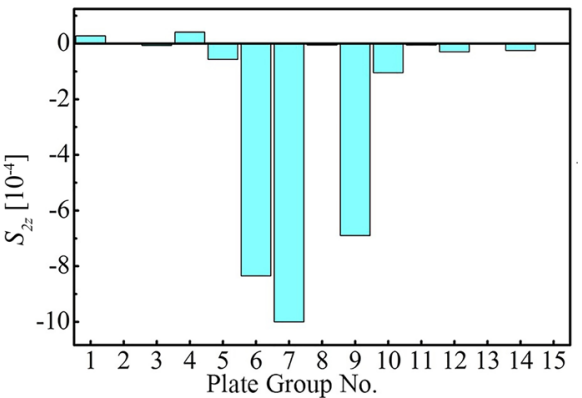

d)

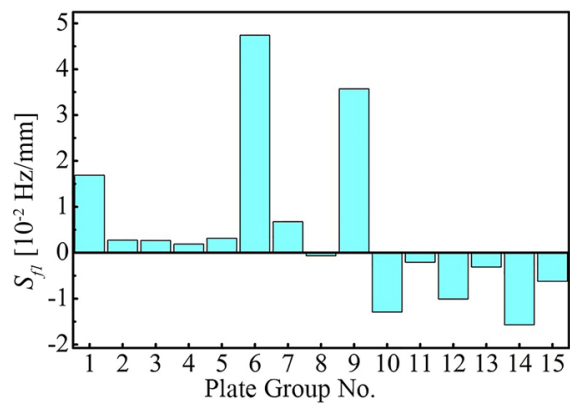

f)

Fig. 3. Sensitivity with respect to thicknesses for: a) total mass, b) compliance, c) displacement sensitivity of node 1 in Z direction, d) displacement sensitivity of node 2 in $Z$ direction, e) displacement sensitivity of node 3 in $Y$ direction, f) first-order frequency sensitivity

Table 4. Performance comparisons between the initial and optimized frame

\begin{tabular}{lccccccc}
\hline & $M[\mathrm{~kg}]$ & $\sigma_{\max }[\mathrm{MPa}]$ & $c[\mathrm{~kJ}]$ & $d_{1 z}[\mathrm{~mm}]$ & $d_{2 z}[\mathrm{~mm}]$ & $d_{3 y}[\mathrm{~mm}]$ & $f_{1}[\mathrm{~Hz}]$ \\
\hline Initial & 45329.96 & 75.53 & 2.195724 & 0.08926 & 0.2767 & 0.06973 & 39.73362 \\
\hline Designed & 39466.08 & 64.49 & 2.134107 & 0.08278 & 0.2729 & 0.06830 & 40.14200 \\
\hline Change & dec. $12.94 \%$ & dec. $14.62 \%$ & dec. $2.81 \%$ & dec. $7.26 \%$ & dec. $1.37 \%$ & dec. $2.05 \%$ & inc. $1.03 \%$ \\
\hline
\end{tabular}

Inc. and dec. are short for increased and decreased, respectively.

which means that the dynamic performance has been improved.

\subsection{Further Discussion}

Modal frequencies of a structure provide useful information about the dynamic behaviour of the system. In fact, in most of the low-frequency vibration problems, the response of the structure is primarily a function of its fundamental frequencies and mode shapes [29]. Generally, it is desirable to increase the modal frequencies of a structure in order to keep out the unwelcome resonance phenomenon. In the previous section, we observed that the first-order modal frequency of the frame could be improved by 
increasing the lower mass and decreasing the upper mass, which can be generalized to more general cases.

A simple method to investigate the vibration characteristics of a continuous system involves replacing the distributed mass or inertia of the system by a finite number of lumped masses, which are assumed to be connected by massless elastic and damping members [30]. In general, dampers mainly affect the attenuation rate of free vibration, and it has little influence on the value of modal frequency. Therefore, to simplify the calculation, a three-lumpedmass model was used to investigate the effect of mass distribution on the modal frequencies of a restrained structure in this study. As shown in Fig. 4, $m_{1}, m_{2}$, and $m_{3}$ denote the mass of the lumped-masses, $k_{1}$, $k_{2}$, and $k_{3}$ indicate the stiffness of the connecting springs, and $x_{1}, x_{2}$, and $x_{3}$ describe the positions of the lumped-masses measured from their respective static equilibrium position.

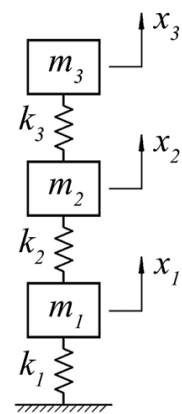

Fig. 4. A three-lumped-mass model

The free vibration equation of the three-lumpedmodel can be expressed as,

$$
\mathbf{m} \mathbf{x}^{\prime \prime}+\mathbf{k x}=\mathbf{0},
$$

where $\mathbf{m}$ and $\mathbf{k}$ are the mass and stiffness matrices, respectively, and are given by,

$$
\begin{gathered}
\mathbf{m}=\left[\begin{array}{ccc}
m_{1} & 0 & 0 \\
0 & m_{2} & 0 \\
0 & 0 & m_{3}
\end{array}\right], \\
\mathbf{k}=\left[\begin{array}{ccc}
k_{1}+k_{2} & -k_{2} & 0 \\
-k_{2} & k_{2}+k_{3} & -k_{3} \\
0 & -k_{3} & -k_{3}
\end{array}\right],
\end{gathered}
$$

and $\mathbf{x}$ and $\mathbf{x} "$ are the displacement and acceleration vectors, respectively, given by,

$$
\begin{aligned}
\mathbf{X} & =\left[\begin{array}{lll}
x_{1} & x_{2} & x_{3}
\end{array}\right]^{T}, \\
\mathbf{X}^{\prime \prime} & =\left[\begin{array}{lll}
x_{1}^{\prime \prime} & x_{2}^{\prime \prime} & x_{3}^{\prime \prime}
\end{array}\right]^{T} .
\end{aligned}
$$

The corresponding characteristic equation of Eq. (12) is,

$$
\left|\mathbf{k}-\omega_{n}{ }^{2} \mathbf{m}\right|=0,
$$

where $\omega_{n}{ }^{2}$ is known as the eigenvalue, and $\omega_{n}$ is the $n^{\text {th }}$ order modal circular frequency of the system.

Assuming that the total mass of the lumpedmasses is unit mass, and each stiffness of the springs is unit stiffness, namely $m_{1}+m_{2}+m_{3}=1$ and $k_{1}=k_{2}=k_{3}=1$, the mass and stiffness matrices can be given by,

$$
\begin{gathered}
\mathbf{m}=\left[\begin{array}{ccc}
m_{1} & 0 & 0 \\
0 & 1-m_{1}-m_{3} & 0 \\
0 & 0 & m_{3}
\end{array}\right], \\
\left(0<m_{1}<1,0<m_{3}<1,0<m_{1}+m_{3}<1\right), \\
\mathbf{k}=\left[\begin{array}{ccc}
2 & -1 & 0 \\
-1 & 2 & -1 \\
0 & -1 & 1
\end{array}\right] .
\end{gathered}
$$

By substituting Eqs. (18) and (19) into Eq. (17), we obtained,

$$
\begin{gathered}
\left(m_{1}^{2} m_{3}+m_{1} m_{3}^{2}-m_{1} m_{3}\right) \cdot\left(\omega_{n}^{2}\right)^{3} \\
+\left(-m_{1}^{2}-2 m_{3}^{2}-m_{1} m_{3}+m_{1}+2 m_{3}\right) \cdot\left(\omega_{n}^{2}\right)^{2} \\
+\left(m_{1}-m_{3}-2\right) \cdot \omega_{n}^{2}+1=0, \\
\left(0<m_{1}<1,0<m_{3}<1,0<m_{1}+m_{3}<1\right) .
\end{gathered}
$$

According to the numerical solution of Eq. (20), a variation of $\omega_{1}$ with the mass distribution is illustrated in Fig. 5a. To reveal the varying pattern of $\omega_{1}$ more clearly, Figs. $5 \mathrm{~b}$ to $\mathrm{d}$ show the variations of $\omega_{1}$ with one lumped-mass fixed and one lumped-mass varying. From Fig. 5b, we can observed that when $m_{1}$ fixed, the higher $m_{2}$ is (or the less $m_{3}$ is), the higher $\omega_{1}$ is. Similar conclusions can be drawn from Figs. $5 \mathrm{c}$ and $\mathrm{d}$. Extending to more general cases, we can conclude that for a constrained structure, material distributing close to the constraint location makes for the increasing of the first-order modal frequency.

Except for $\omega_{1}$, we also investigated the variation of $\omega_{2}$ and $\omega_{3}$ with the mass distribution, as shown in Fig. 6. Two fascinating phenomena can be observed: 1) when the gross mass approaches to concentrating upon an arbitrarily lumped mass, $\omega_{2}$ approaches to a maximum value, except which $\omega_{2}$ has no obvious changes; 2) when the mass of an arbitrarily lumped mass approaches to zero, $\omega_{3}$ approaches to a maximum value, except which $\omega_{3}$ has no obvious changes. 


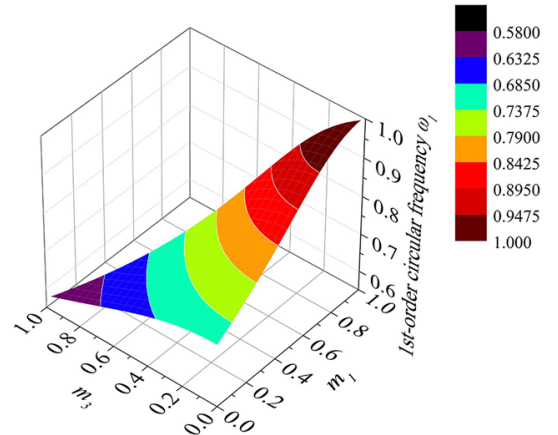

a)

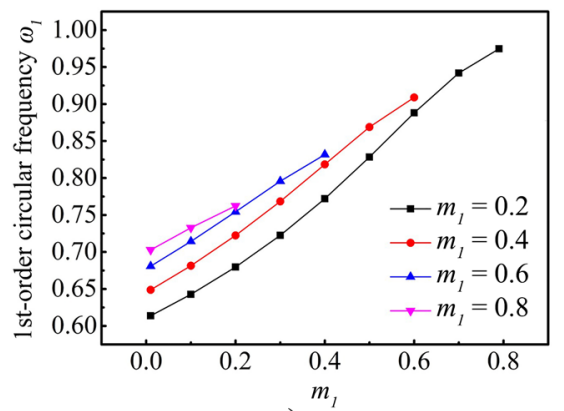

c)

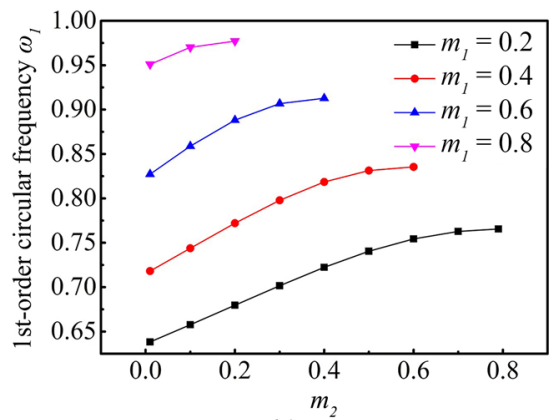

b)

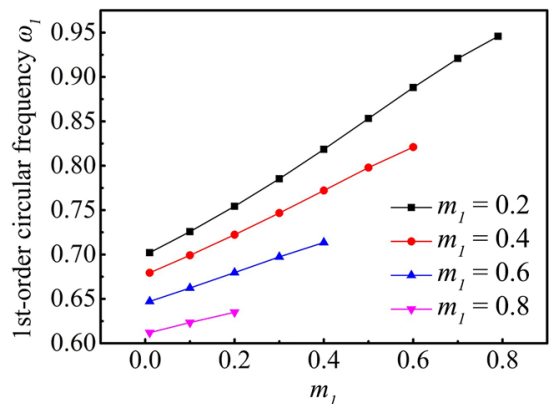

d)

Fig. 5. a) Variation of $\omega_{1}$ with $m_{1}$ and $m_{3}$ varying, b) variation of $\omega_{1}$ with $m_{1}$ fixed and $m_{2}$ varying, c) variation of $\omega_{1}$ with $m_{2}$ fixed and $m_{1}$ varying, d) variation of $\omega_{1}$ with $m_{3}$ fixed and $m_{1}$ varying

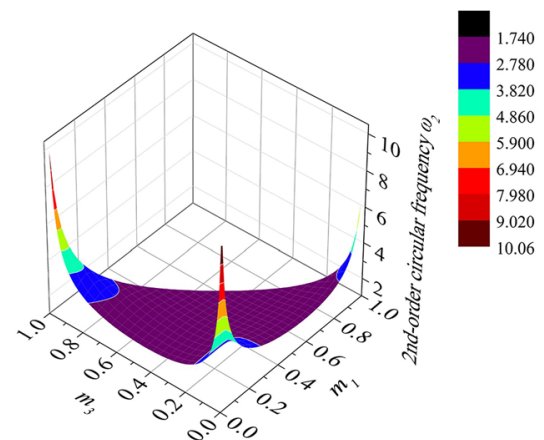

a)

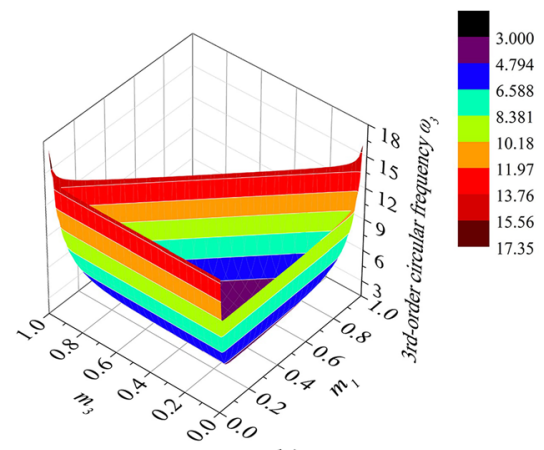

b)

Fig. 6. a) Variation of $\omega_{2}$ with $m_{1}$ and $m_{3}$ varying, and b) variation of $\omega_{3}$ with $m_{1}$ fixed and $m_{2}$ varying

\section{CONCLUSIONS}

This study aims to analyse and optimize the 12,000 $\mathrm{kN}$ fine-blanking press frame by adopting sensitivity analysis and size optimization. We also analysed the influence of the material distribution on the dynamic structure performance. The main contributions and conclusions of this paper include:

1) A shell element model was set up to analyse and optimize the fine-blanking press frame. Mathematical formulas of the sensitivity analysis and size optimization for the fine-blanking press frame were established.

2) The plate thicknesses of the frame were optimized by adopting sensitivity analysis and size optimization. The FEA result of solid element model showed that the mass of the frame was reduced by $12.94 \%$, while the structure strength had an acceptable reduction and the global stiffness, local stiffness and dynamic performance have been improved.

3) The effect of the material distribution on the dynamic performance of a constrained structure 
was investigated with a three-lumped-mass model. The result indicated that material distributing close to the constraint location makes for the increasing of the modal frequencies.

\section{ACKNOWLEDGEMENTS}

The authors would like to thank the 111 Project (B17034) and the National Natural Science Foundation of China (Grant No. 51375356) for the supports given to this research. The authors also gratefully acknowledge the mechanical engineers of Huangshi Huali Metal Forming Machine Tool Inc., who provided useful data and helpful suggestions.

\section{REFERENCES}

[1] Liu, Y.X., Cheng, T., Hua, L., Mao, H.J. (2017). Research on the effect of ultrasonic vibration on the roll-over during the fine blanking process. Journal of Mechanical Science and Technology, vol. 31, no. 2, p. 835-843, D0l:10.1007/s12206017-0135-z.

[2] Strano, M., Monno, M., Rossi, A. (2013). Optimized design of press frames with respect to energy efficiency. Journal of Cleaner Production, vol. 41, p. 140-149, D0l:10.1016/j. jclepro.2012.10.017.

[3] Bendsøe, M., Sigmund, O. (2003). Topology Optimization: Theory, Methods and Applications, Springer, Berlin.

[4] Yang, K.K., Fernandez, E., Niu, C., Duysinx, P. Zhu, J.H., Zhang, W.H. (2019). Note on spatial gradient operators and gradient-based minimum length constraints in SIMP topology optimization. Structural and Multidisciplinary Optimization, vol. 60, p. 393-400, DI0:10.1007/s00158-019-02269-9.

[5] Sethian, J.A., Wiegmann, A. (2000). Structural boundary design via level set and immersed interface methods. Journal of Computational Physics, vol. 163, no. 2, p. 489-528, DOl:10.1006/jcph.2000.6581.

[6] Khan, W., Islam, S.U., Ullah, B. (2019). Structural optimization based on meshless element free Galerkin and level set methods. Computer Methods in Applied Mechanics and Engineering, vol. 334, p. 144-163, D0I:10.1016/j. cma.2018.09.024.

[7] Xie, Y.M., Steven, G.P. (2012). Evolutionary Structural Optimization, Springer, Berlin.

[8] Li, B.T., Liu, H.L., Yang, Z.H., Zhang, J.H. (2019). Stiffness design of plate/shell structures by evolutionary topology optimization. Thin-Walled Structures, vol. 141, p. 232-250, DOl:10.1016/j.tws.2019.04.012.

[9] Hetrick, J.A., Kota, S. (1999). An energy formulation for parametric size and shape optimization of compliant mechanisms. Journal of Mechanical Design, vol. 121, no. 2, p. 229-234, Dol:10.1115/1.2829448.

[10] Kaveh. A., Zolghadr, A. (2011). Shape and size optimization of truss structures with frequency constraints using enhanced charged system search algorithm. Asian Journal of Civil Engineering (Building and Hosing), vol. 12, no. 4, p. 487-509.
[11] Hojjat, M., Stavropoulou, E., Bletzinger, K.U. (2014). The vertex morphing method for node-based shape optimization. Computer Methods in Applied Mechanics Engineering, vol. 268, p. 494-513, Dol:10.1016/J.cma.2013.10.015.

[12] Ma, H.F., Wang, J.X., Lu, Y.N., Guo, Y.W. (2019). Lightweight design of turnover frame of bridge detection vehicle using topology and thickness optimization. Structural and Multidisciplinary Optimization, vol. 59, no. 3, p. 1007-1019, DOI:10.1007/s00158-018-2113-1.

[13] Li, C., Kim, I.Y., Jeswiet, J. (2014). Conceptual and detailed design of an automotive engine cradle by using topology, shape, and size optimization. Structural and Multidisciplinary Optimization, vol. 51, no. 2, p. 547-564, D0l:10.1007/s00158014-1151-6.

[14] Li, A., Liu, C., Feng, S. (2018). Topology and thickness optimization of an indenter under stress and stiffness constraints. Journal of Mechanical Science and Technology, vol. 31, no. 1, p. 211-222, Dol:10.1007/s12206-017-1222-x.

[15] Cheng, Q., Zhan, C.P., Liu, Z.F., Zhao, Y.S., Gu, P.H. (2015). Sensitivity-based multidisciplinary optimal design of a hydrostatic rotary table with particle swarm optimization. Strojniški vestnik - Journal of Mechanical Engineering, vol. 61, no. 7-8, p. 432-447, D0l:10.5545/sv-jme.2015.2478.

[16] Kim, I.Y., Kwak, B.M. (1993). Shape design sensitivity analysis and optimization of general shape arches. Computers \& Structures, vol. 48, no. 6, p. 1025-1031, Dol:10.1016/00457949(93)90437-I.

[17] Zuo, W.J., Huang, K., Bai, J.T., Guo, G.K. (2016). Sensitivity reanalysis of vibration problem using combined approximations method. Structural and Multidisciplinary Optimization, vol. 55, no. 4, p. 1399-1405, Dol:10.1007/ s00158-016-1586-z.

[18] Strano, M., Monno, M., Rossi, A. (2012). Optimization of prestressed press frames. Key Engineering Materials, vol. 504506, p. 625-630, D0I:10.4028/www.scientific.net/kem.504506.625.

[19] Trebuňa, F., Šimčák, F., Bocko, J., Trebuňa, P., Pástor, M., Šarga, P. (2011). Analysis of crack initiation in the press frame and innovation of the frame to ensure its further operation. Engineering Failure Analysis, vol. 18, no. 1, p. 244-255, DOI:10.1016/j.engfailanal.2010.09.004.

[20] Zhang, W.W., Wang, X.S., Wang, Z.R., Yuan, S.J., He, Z.B., Liu, G., Dai, K. (2014). Mechanical analysis on the cylinder-crown integrated hydraulic press with a hemispherical cylinder. Proceedings of the Institution of Mechanical Engineers, Part C: Journal of Mechanical Engineering Science, vol. 229, no. 3, p. 407-416, DOI:10.1177/0954406214537800.

[21] Wang, W.J., Lin, F., Zhang, L., Wang, X. (2016). Experimental study and finite element analysis on the frame of multi-directional forging press. Proceedings of the Institution of Mechanical Engineers, Part B: Journal of Engineering Manufacture, vol. 231, no. 12, p. 2112-2122, DOI:10.1177/0954405415624658.

[22] Glebov, A.O., Karpov, S.V., Malygin E.N. (2020). Comparison of topological optimization methods on the example of column press traverse. IOP Conference Series: Materials Science and Engineering, vol. 709, no. 2, Dol:10.1088/1757. 899X/709/2/022027. 
[23] Lan, J., Hu, J.W., Song, C.P., Hua, L., Zhao, Y.M. (2011). Modeling and optimization of a $10000 \mathrm{KN}$ fine blanking press frame. Remote Sensing, Environment and Transportation Engineering (RSETE), p. 8353-8357, D0l:10.1109/ rsete.2011.5964103.

[24] Zhao, X.H., Liu, Y.X., Hua, L., Mao, H.J. (2016). Finite element analysis and topology optimization of a $12000 \mathrm{kN}$ fine blanking press frame. Structural and Multidisciplinary Optimization, vol. 54, no. 2, p. 375-389, D0l:10.1007/s00158-016-1407-4.

[25] Wang, X., Mai, Y.F. (2009). The choice of element type in FEA. Mechanical Research \& Application, vol. 22, no, 6, p. 43-46, D0l:1006-4414(2009)06-0043-04. (in Chinese)

[26] Altair Optistruct (2011). OptiStruct 11.0 user manual. Altair Inc., Troj

[27] Xiao, D.H., Zhang, H., Liu, X.D., He, T., Shan, Y.C. (2014). Novel steel wheel design based on multi-objective topology optimization. Journal of Mechanical Science and Technology, vol. 28 , no. 3, p. 1007-1016, D0l:10.1007/s12206-013-11748.

[28] Xie, G.L., Dong, Y.J., Zhu, J., Sheng, Z.Q. (2020). Topology optimization design of hydraulic valve blocks for additive manufacturing. Proceedings of the Institution of Mechanical Engineers, Part C: Journal of Mechanical Engineering Science, vol. 234, no. 10, p. 1899-1912, DOI:10.1177/0954406220902166.

[29] Grandhi, R.V., Venkayya, V.B. (1988). Structural optimization with frequency constraints. AIAA Journal, vol. 26, no. 7, p. 858-866, D0l:10.2514/3.9979.

[30] Rao, S.S., Yap, F.F. (2011). Mechanical Vibrations. Prentice Hall, Upper Saddle River. 